\title{
An Algorithm for Obtaining an Orthogonal Set of Individual Degrees of Freedom for Error
}

\author{
Joseph M. Cameron
}

(November 20, 1962)

\begin{abstract}
This note presents an algorithm based on the Gram-Schmidt orthonormalization procedure for producing the coefficients of linear combinations of observations which can be used for computing an orthogonal set of individual degrees of freedom for error from a set of observations.
\end{abstract}

In the analysis of data from designed experiments it is becoming common to compute the deviations between the observed and predicted values. Because of the correlation among these residuals it is sometimes easier to interpret a set of independent individual degrees of freedom, particularly if the residuals are to be used to study the state of statistical control of a measurement process. This note presents an algorithm for producing such an orthogonal set.

Let the observations $Y_{1}, Y_{2}, \ldots Y_{n}$ have expected values

$$
\begin{aligned}
& E\left(Y_{1}\right)=\beta_{0} X_{01}+\beta_{1} X_{11}+\ldots+\beta_{k} X_{k 1} \\
& E\left(Y_{2}\right)=\beta_{0} X_{02}+\beta_{1} X_{12}+\ldots++\beta_{k} X_{k 2} \\
& \cdot . \\
& E\left(Y_{n}\right)=\beta_{0} X_{0 n}+\beta_{1} X_{1 n}+\ldots+\beta_{k} X_{k n}
\end{aligned}
$$

which can be written in matrix notation as

$$
E(y)=X \beta
$$

where $y$ is the vector of observations, $X$ the array of $X_{i j}$ 's and $\beta$ a vector of parameters.

The deviation, $\delta_{i}$, between the observed and predicted value is then

$$
\delta_{i}=y_{i}-\hat{\beta}_{0} X_{0 i}-\hat{\beta}_{1} X_{1 i}-\ldots-\hat{\beta}_{k} X_{i i}
$$

or in matrix form

$$
\delta=y-X \hat{\beta}
$$

where $\hat{\beta}$ is the vector of estimates of the parameters of the system. Noting that the $\hat{\beta}_{i}$ are functions of the observations each $\delta_{i}$ can also be written as a function of the observation, say

$$
\delta=A y
$$

To say that there are $m$ degrees of freedom for error is to say that $A$ has rank $m$ or that the rows of $A$ are linear combinations of a set of $m$ orthogonal rows. These $m$ orthogonal rows give the coefficients necessary for computing the individual degrees of freedom for error.

The orthogonalization can be carried out by an application of the Gram-Schmidt process, ${ }^{1}$

1 Philip Davis and Philip Rabinowitz. A multiple purpose orthonormalizing code and its uses, Journal of the Association for Comp uting Machinery 1, 183-191 (1954). 
a modification of which follows. The deviations can be written as function of the observations as follows:

$$
\begin{aligned}
& \delta_{1}=a_{11} y_{1}+a_{12} y_{2}+\ldots+a_{1 n} y_{n} \\
& \delta_{2}=a_{21} y_{1}+a_{22} y_{2}+\ldots+a_{2 n} y_{n} \\
& \ldots \\
& \delta_{n}=a_{n 1} y_{1}+a_{n 2} y_{2}+\ldots+a_{n n} y_{n} .
\end{aligned}
$$

Consider now the vectors

$$
\begin{aligned}
& a_{1}=\left(a_{11}, a_{12}, \ldots, a_{1 n}\right) \\
& a_{2}=\left(a_{21}, a_{22}, \ldots, a_{2 n}\right) \\
& \ldots \\
& a_{n}=\left(a_{n 1}, a_{n 2}, \ldots, a_{n n}\right)
\end{aligned}
$$

and denote the sums of squares and cross products as follows

$$
\begin{aligned}
& \left(a_{k} \cdot a_{k}\right)=\sum_{i=1}^{n} a_{k i}^{2} \\
& \left(a_{k} \cdot a_{l}\right)=\sum_{i=1}^{n} a_{k i} a_{l i} \cdot
\end{aligned}
$$

Form the vectors

$$
\begin{aligned}
& b_{1}= \\
& b_{2}=\left(a_{1} \cdot a_{1}\right) a_{2}-\left(a_{1} \cdot a_{2}\right) a_{1} \\
& b_{3}=\left(a_{1} \cdot a_{1}\right) a_{3}-\left(a_{1} \cdot a_{3}\right) a_{1} \\
& \dot{.} \cdot \\
& b_{n}=\left(a_{1} \cdot a_{1}\right) a_{n}-\left(a_{1} \cdot a_{n}\right) a_{1}
\end{aligned}
$$

where the vectors $b_{i}$ are the vector sums of the indicated vectors, e.g., the elements of $b_{2}$ are

$$
\left(a_{1} \cdot a_{1}\right) a_{21}-\left(a_{1} \cdot a_{2}\right) a_{11},\left(a_{1} \cdot a_{1}\right) a_{22}-\left(a_{1} \cdot a_{2}\right) a_{12}, \ldots,\left(a_{1} \cdot a_{1}\right) a_{2 n}-\left(a_{1} \cdot a_{2}\right) a_{1 n} .
$$

Next form

$$
\begin{array}{lr}
c_{1}= & b_{1} \\
c_{2}= & b_{2} \\
c_{3}=\left(b_{2} \cdot b_{2}\right) b_{3}-\left(b_{2} \cdot b_{3}\right) b_{2} \\
c_{4}=\left(b_{2} \cdot b_{2}\right) b_{4}-\left(b_{2} \cdot b_{4}\right) b_{2} \\
\cdot . \\
c_{n}=\left(b_{2} \cdot b_{2}\right) b_{n}-\left(b_{2} \cdot b_{n}\right) b_{2}
\end{array}
$$

Then

$$
\begin{array}{lr}
d_{1}= & c_{1} \\
d_{2}= & c_{2} \\
d_{3}=r & c_{3} \\
d_{4}=\left(c_{3} \cdot c_{3}\right) c_{4}-\left(c_{3} \cdot c_{4}\right) c_{3} \\
d_{5}=\left(c_{3} \cdot c_{3}\right) c_{5}-\left(c_{3} \cdot c_{5}\right) c_{3}
\end{array}
$$

If in the process one of the vectors becomes all zeros, it should be transferred to the end and the vectors renumbered accordingly.

This process is continued for $m$ steps at which point the last $n-m$ vectors will be zero vectors and the first $m$ will be the desired orthogonal set. It is easy to verify that at the $k$ th stage the first $(k-1)$ vectors are mutually orthogonal and orthogonal to all vectors below. 
To illustrate, consider the design where all differences among four objects are measured i.e.,

$$
\begin{aligned}
& E\left(Y_{1}\right)=\beta_{1}-\beta_{2} \\
& E\left(Y_{2}\right)=\beta_{1}-\beta_{3} \\
& E\left(Y_{3}\right)=\beta_{1}-\beta_{4} \\
& E\left(Y_{4}\right)=\beta_{2}-\beta_{3} \\
& E\left(Y_{5}\right)=\beta_{2}-\beta_{4} \\
& E\left(Y_{6}\right)=\beta_{3}-\beta_{4} .
\end{aligned}
$$

The following table shows the estimates of the $\beta$ 's under the assumption that $\Sigma \beta=0$.

$\begin{array}{crrrrrr} & Y_{1} & Y_{2} & Y_{3} & Y_{4} & Y_{5} & Y_{6} \\ 4 \hat{\beta}_{1} & 1 & 1 & 1 & 0 & 0 & 0 \\ 4 \hat{\beta}_{2} & -1 & 0 & 0 & 1 & 1 & 0 \\ 4 \hat{\beta}_{3} & 0 & -1 & 0 & -1 & 0 & 1 \\ 4 \hat{\beta}_{4} & 0 & 0 & -1 & 0 & -1 & -1 \\ ------------ & -- & - & --- \\ 4 \delta_{1}=4 Y_{1}-4\left(\hat{\beta}_{1}-\hat{\beta}_{2}\right) & 2 & -1 & -1 & 1 & 1 & 0 \\ 4 \delta_{2}=4 Y_{2}-4\left(\hat{\beta}_{1}-\hat{\beta}_{3}\right) & -1 & 2 & -1 & -1 & 0 & 1 \\ 4 \delta_{3}=4 Y_{3}-4\left(\hat{\beta}_{1}-\hat{\beta}_{4}\right) & -1 & -1 & 2 & 0 & -1 & -1 \\ 4 \delta_{4}=4 Y_{4}-4\left(\hat{\beta}_{2}-\hat{\beta}_{3}\right) & 1 & -1 & 0 & 2 & -1 & 1 \\ 4 \delta_{5}=4 Y_{5}-4\left(\hat{\beta}_{2}-\hat{\beta}_{4}\right) & 1 & 0 & -1 & -1 & 2 & -1 \\ 4 \delta_{6}=4 Y_{6}-4\left(\hat{\beta}_{3}-\hat{\beta}_{4}\right) & 0 & 1 & -1 & 1 & -1 & 2\end{array}$

From this table one has, for example:

$$
\begin{aligned}
& \hat{\beta}_{1}=\frac{1}{4}\left(Y_{1}+Y_{2}+Y_{3}\right) \\
& \delta_{1}=\frac{1}{4}\left(2 Y_{1}-Y_{2}-Y_{3}+Y_{4}+Y_{5}\right)
\end{aligned}
$$

The matrix $A$ is then

$$
\begin{aligned}
& a_{1}=\left(\begin{array}{cccccc}
2 & -1 & -1 & 1 & 1 & 0
\end{array}\right) \quad\left(a_{1} a_{1}\right)=8 \\
& a_{2}=\left(\begin{array}{llllll}
-1 & 2 & -1 & -1 & 0 & 1
\end{array}\right) \quad\left(a_{1} a_{2}\right)=-4 \\
& a_{3}=\left(\begin{array}{llllll}
-1 & -1 & 2 & 0 & -1 & -1
\end{array}\right) \quad\left(a_{1} a_{3}\right)=-4 \\
& a_{4}=\left(\begin{array}{llllll}
1 & -1 & 0 & 2 & -1 & 1
\end{array}\right) \quad\left(a_{1} a_{4}\right)=4 \\
& a_{5}=\left(\begin{array}{llllll}
1 & 0 & -1 & -1 & 2 & -1
\end{array}\right) \quad\left(a_{1} a_{5}\right)=4 \\
& a_{6}=\left(\begin{array}{llllll}
0 & 1 & -1 & 1 & -1 & 2
\end{array}\right) \quad\left(a_{1} a_{6}\right)=0
\end{aligned}
$$

The first step gives

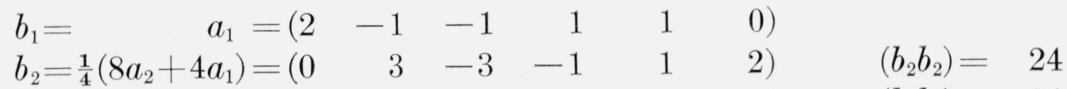

$$
\begin{aligned}
& b_{3}=\frac{1}{4}\left(8 a_{3}+4 a_{1}\right)=\left(\begin{array}{llllll}
0 & -3 & 3 & 1 & -1 & -2
\end{array}\right) \quad\left(b_{2} b_{3}\right)=-24 \\
& b_{4}=\frac{1}{4}\left(8 a_{4}-4 a_{1}\right)=\left(\begin{array}{llllll}
0 & -1 & 1 & 3 & -3 & 2
\end{array}\right) \quad\left(b_{2} b_{4}\right)=-8 \\
& b_{5}=\frac{1}{4}\left(8 a_{5}-4 a_{1}\right)=\left(\begin{array}{llllll}
0 & 1 & -1 & -3 & 3 & -2
\end{array}\right) \quad\left(b_{2} b_{5}\right)=8 \\
& b_{6}=\frac{1}{8}\left(\begin{array}{llllll}
8 a_{6} & )
\end{array}\right)=\left(\begin{array}{llllll}
0 & 1 & -1 & 1 & -1 & 2
\end{array}\right) \quad\left(b_{2} b_{6}\right)=\quad 8 .
\end{aligned}
$$

The vectors $b_{2}, b_{3}, \ldots$ are multiplied by factors (e.g., $1 / 4,1 / 4$, . .) to keep the numbers conveniently small. 
The second step gives

$$
\begin{aligned}
& c_{1}=\quad b_{1}=\left(\begin{array}{llllll}
2 & -1 & -1 & 1 & 1 & 0
\end{array}\right) \\
& c_{2}=\quad b_{2}=\left(\begin{array}{llllll}
0 & 3 & -3 & -1 & 1 & 2
\end{array}\right) \\
& c_{3}=24 b_{3}+24 b_{2}=\left(\begin{array}{llllll}
0 & 0 & 0 & 0 & 0 & 0
\end{array}\right) \\
& c_{4}=\frac{1}{64}\left(24 b_{4}+8 b_{2}\right)=\left(\begin{array}{llllll}
0 & 0 & 0 & 1 & -1 & 1
\end{array}\right) \\
& c_{5}=\frac{1}{64}\left(24 b_{5}-8 b_{2}\right)=\left(\begin{array}{llllll}
0 & 0 & 0 & -1 & 1 & -1
\end{array}\right) \\
& c_{6}=\frac{1}{32}\left(24 b_{6}-8 b_{2}\right)=\left(\begin{array}{llllll}
0 & 0 & 0 & 1 & -1 & 1
\end{array}\right)
\end{aligned}
$$

Vector $c_{3}$ is transferred to the end to give

$$
\begin{aligned}
& c_{1}=\left(\begin{array}{llllll}
2 & -1 & -1 & 1 & 1 & 0
\end{array}\right) \\
& c_{2}=\left(\begin{array}{llllll}
0 & 3 & -3 & -1 & 1 & 2
\end{array}\right) \\
& c_{3}=\left(\begin{array}{llllll}
0 & 0 & 0 & 1 & -1 & 1
\end{array}\right) \quad\left(c_{3} c_{3}\right)=3 \\
& c_{4}=\left(\begin{array}{llllll}
0 & 0 & 0 & -1 & 1 & -1
\end{array}\right) \quad\left(c_{3} c_{4}\right)=-3 \\
& c_{5}=\left(\begin{array}{llllll}
0 & 0 & 0 & 1 & -1 & 1
\end{array}\right) \quad\left(c_{3} c_{5}\right)=3 \\
& c_{6}=\left(\begin{array}{llllll}
0 & 0 & 0 & 0 & 0 & 0
\end{array}\right) \quad\left(c_{3} c_{6}\right)=0
\end{aligned}
$$

The next stage gives

$$
\begin{aligned}
& d_{1}=\quad c_{1}=\left(\begin{array}{llllll}
2 & -1 & -1 & 1 & 1 & 0
\end{array}\right) \\
& d_{2}=\quad c_{2}=\left(\begin{array}{llllll}
0 & 3 & -3 & -1 & 1 & 2
\end{array}\right) \\
& d_{3}=\quad c_{3}=\left(\begin{array}{llllll}
0 & 0 & 0 & 1 & -1 & 1
\end{array}\right) \\
& d_{4}=3 c_{4}+3 c_{3}=\left(\begin{array}{llllll}
0 & 0 & 0 & 0 & 0 & 0
\end{array}\right) \\
& d_{5}=3 c_{5}-3 c_{3}=\left(\begin{array}{llllll}
0 & 0 & 0 & 0 & 0 & 0
\end{array}\right) \\
& d_{6}=\quad 0=\left(\begin{array}{llllll}
0 & 0 & 0 & 0 & 0 & 0
\end{array}\right)
\end{aligned}
$$

with $d_{1}, d_{2}, d_{3}$, being the desired set of orthogonal vectors.

It is worth noting that one need not start with the deviations, but may begin with any set of linear functions having zero expectation and rank equal to the number of degrees of freedom for error. For the example the three vectors below could have been used.

$$
\begin{aligned}
& a_{1}=\left(\begin{array}{llllll}
1 & -1 & 0 & 1 & 0 & 0
\end{array}\right) \\
& a_{2}=\left(\begin{array}{llllll}
0 & 0 & 0 & 1 & -1 & 1
\end{array}\right) \\
& a_{3}=\left(\begin{array}{llllll}
0 & 1 & -1 & 0 & 0 & 1
\end{array}\right)
\end{aligned}
$$

The steps in the orthogonalization are

$$
\begin{aligned}
& \begin{array}{llllllll}
b_{1}= & a_{1}=; & 1 & -1 & 0 & 1 & 0 & 0)
\end{array} \\
& b_{2}=3 a_{2}-a_{1}=\left(\begin{array}{llllll}
-1 & 1 & 0 & 2 & -3 & 3
\end{array}\right) \\
& b_{3}=3 a_{3}+a_{1}=\left(\begin{array}{llllll}
1 & 2 & -3 & 1 & 0 & 3
\end{array}\right) \\
& c_{1}=\quad b_{1}=\left(\begin{array}{rrrrrr}
1 & -1 & 0 & 1 & 0 & 0
\end{array}\right) \\
& c_{2}=\quad b_{2}=\left(\begin{array}{llllll}
-1 & 1 & 0 & 2 & -3 & 3
\end{array}\right) \\
& c_{3}=\frac{1}{36}\left(24 b_{3}-12 b_{2}\right)=\left(\begin{array}{lllllll}
1 & 1 & -2 & 0 & 1 & 1
\end{array}\right) \text {. }
\end{aligned}
$$

(Paper 67B1-89) 